\title{
Mid-infrared wavelength division (de)multiplexer using an interleaved angled multimode interferometer on the silicon-on-insulator platform
}

\author{
Y. Hu, ${ }^{*}$ T. Li, D. J. Thomson, X. Chen, J. Soler Penades, A. Z. Khokhar, C. J. Mitchell, \\ G. T. Reed, and G. Z. Mashanovich \\ Optoelectronics Research Centre, University of Southampton, Southampton SO17 1BJ, UK \\ *Corresponding author: y.hu@soton.ac.uk
}

Received November 22, 2013; revised January 28, 2014; accepted January 29, 2014; posted January 31, 2014 (Doc. ID 201891); published March 5, 2014

\begin{abstract}
A low-cost and high-performance wavelength division (de)multiplexing structure in the mid-IR wavelength range is demonstrated on the silicon-on-insulator platform using an interleaved angled multimode interferometer (AMMI). As compared to a single AMMI, the channel count was doubled and the channel spacing halved with negligible extra insertion loss and crosstalk and with only a slight increase in device footprint. The device requires only single lithography and etching steps for fabrication. Potential is also shown for achieving improved performance with further optimized design. (C) 2014 Optical Society of America

OCIS codes: (230.3120) Integrated optics devices; (230.7390) Waveguides, planar; (230.7408) Wavelength filtering devices; (250.5300) Photonic integrated circuits; (350.2460) Filters, interference.

http://dx.doi.org/10.1364/OL.39.001406
\end{abstract}

As a mature material platform for near-IR silicon photonics, the silicon-on-insulator (SOI) platform has also been demonstrated to be suitable for the fabrication of low-loss mid-IR waveguides [1,2]. A variety of SOI-based mid-IR passive components have been successfully demonstrated including power splitters/combiners, arrayed waveguide gratings (AWGs) [3], planar concave gratings (PCGs) [4], angled multimode interferometers (AMMIs) [5], etc. The performance of those components is close to or as good as the counterparts in the near-IR range. There is still a great potential to boost their performance by optimizing the structural parameters of the SOI platform [e.g., the silicon overlayer thickness or the buried oxide (BOX) thickness] or the design parameters of the components (e.g., the waveguide width, bending radii, etch depth, etc.). The major concern for the design of mid-IR SOI waveguides is the suppression of the loss resulting from the absorption and leakage through the BOX layer and the scattering at the $\mathrm{Si} / \mathrm{SiO}_{2}$ interface. As a rule of thumb for the design of mid-IR SOI waveguide components, the SOI platform should have a thick enough silicon overlayer and BOX layer so that the fundamental vertical mode of the waveguide has a field intensity close to zero at the $\mathrm{Si} / \mathrm{SiO}_{2}$ interface; as a result, the effect of surface scattering, $\mathrm{SiO}_{2}$ absorption, and light tunneling from the silicon overlayer to the substrate are kept at a low level.

Mid-IR wavelength division multiplexing (WDM) devices have potential application for sensing and telecommunications [6,7]. Recently, we have demonstrated a 4-channel AMM̄I WDM device at the near-IR C-band [8] and an AMMI triplexer at the mid-IR range [5]. The relatively large waveguide dimensions of the AMMI result in a low insertion loss and ease of fabrication. However, they limit the channel count and result in large channel spacing in a single AMMI device. To overcome this limitation, we have demonstrated an interleaved AMMI device comprising two AMMIs and one MZI in the near-IR wavelength range, which has doubled the channel count and halved the channel spacing compared to a single AMMI device. The design was optimized so that the extra insertion loss and crosstalk are kept low. Here, we report an interleaved AMMI device in the mid-IR wavelength range with further suppressed insertion loss and crosstalk, making the interleaved device a further step toward practical applications.

The devices were designed to operate at a wavelength window between 3.725 and $3.81 \mu \mathrm{m}$, where we could use a tunable mid-IR quantum cascade laser (QCL) from daylight solution. Figure 1(a) shows the schematic drawing of a single 3-channel AMMI. Unlike a conventional multimode interferometer (MMI), the input/output waveguides access the multimode waveguide at a tilted angle, $\theta_{t}$, from its side walls. The positions of the output waveguides were designed using the self-imaging condition of an MMI, given by [9]

$$
L_{i}=\frac{4 n_{\mathrm{eff}, \mathrm{AMMI}} W_{\mathrm{AMMI}}^{2}}{\lambda_{i}} \quad(i=1,2,3 \ldots)
$$

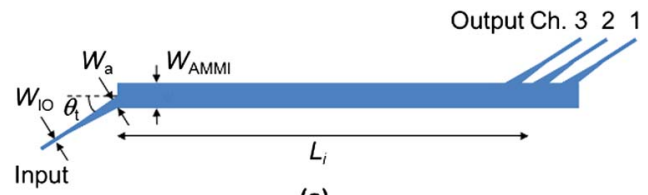

(a)

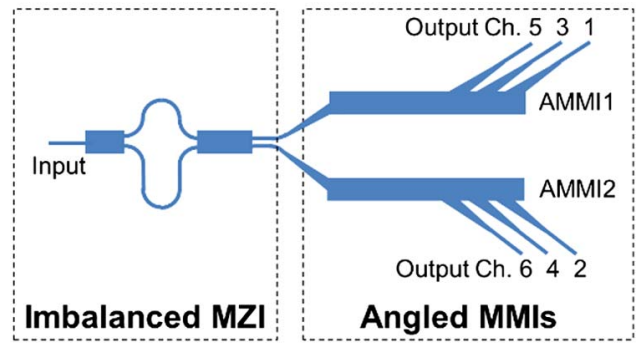

(b)

Fig. 1. Design of (a) a single 3-channel AMMI and (b) an interleaved 6-channel AMMI. 




Fig. 2. Simulated light propagation pattern in a mid-IR AMMI.

where $\lambda_{i}$ is the operating wavelength of the $i$ th channel; $n_{\text {eff,AMMI }}$ is the effective index of the fundamental mode in the multimode waveguide of the AMMI; $W_{\text {AMMI }}$ is the width of the multimode waveguide; and $L_{i}$ is the axial distance between the access points of the input waveguide and the $i$ th output waveguide. The input/output waveguides' widths are tapered from a single-mode width $W_{\text {IO }}$ up to $W_{a}$ before accessing the multimode waveguide (Fig. 1), so that as the light first enters the multimode waveguide its diffraction angle, $\theta_{d}$, is small and the light propagation can be simply treated using ray optics (Fig. 2). In this way, there is a window on the side wall of the multimode waveguide where the light intensity tends to be zero, and it can be used as the access window of the output waveguides of the other channels. Using the ray optics geometry and the diffraction relation

$$
\theta_{d}=C \frac{\lambda_{a}}{W_{a}}
$$

the maximum number of channels for a single AMMI device, $N_{\max }$, is given by

$$
N_{\max }=\frac{2 W_{\mathrm{AMMI}} \theta_{d} \cos \theta_{t}}{C \lambda_{a}}
$$

and the minimum channel spacing, $\Delta \lambda_{\min }$, is given by

$$
\Delta \lambda_{\min }=\frac{4 C \lambda_{a} n_{\mathrm{eff}, \mathrm{AMMI}} W_{\mathrm{AMMI}}^{2}}{L_{a}^{2} \theta_{d} \sin \theta_{t}},
$$

where $C$ is a constant correction factor, $\lambda_{a}$ is the average wavelength of all the channels, and $L_{a}$ is the average axial distance between the input and all the output channels' access points on the multimode waveguide. The temperature sensitivity of AMMI can be derived from Eq. (1) and is given by

$$
\frac{d \lambda_{a}}{d T}=\frac{\lambda_{a}}{n_{\mathrm{eff}, \mathrm{AMMI}}} \frac{d n_{\mathrm{eff}, \mathrm{AMMI}}}{d T} .
$$

From Eqs. (3)-(5), it is evident that the longer the operating wavelength, the fewer channels and the larger wavelength channel spacing are allowed in the AMMI structure, and the larger temperature sensitivity is expected. Previously, we demonstrated an AMMI triplexer with a channel spacing as large as $30 \mathrm{~nm}$ in the mid-IR wavelength range [5]. Further increase of channel count and reduction of channel spacing in a single AMMI could significantly deteriorate the performance of the WDM device, i.e., higher insertion loss and crosstalk.
As shown in our previous work [10], $N_{\max }$ can be doubled and $\Delta \lambda_{\min }$ can be halved in the near-IR C-band when an interleaved device is used. We designed a counterpart of the interleaved angled multimode interferometer (IAMMI) with optimized parameters in the mid-IR wavelength range [Fig. 1(b)]. It is composed of two parts, an imbalanced Mach-Zehnder interferometer (MZI) and two 3-channel AMMIs. The free spectral range (FSR) of the MZI, $\Delta \lambda_{\text {MZI }}$, and the channel spacing of the two AMMIs, $\Delta \lambda_{\text {AMMI }}$, should be equal, and the peak transmission wavelengths of the output channels in the AMMIs $\left(\lambda_{1}-\lambda_{6}\right)$ are determined by the self-imaging condition in Eq. (1). These wavelengths should also satisfy the interleaving condition

$$
\lambda_{i}=\frac{2 n_{g, \mathrm{MZI}} \times \Delta L_{\mathrm{MZI}}}{m+i} \quad(i=1, \ldots, 6),
$$

where $m$ is an integer, $n_{g, \mathrm{MZI}}$ is the average group index of the MZI arm waveguides at wavelengths from $\lambda_{1}$ to $\lambda_{6}$, and $\Delta L_{\text {MZI }}$ is the arm length difference of the imbalanced MZI. Therefore, the whole structure is arranged in a way that the interleaved resonant wavelengths of the MZI passing through the upper and lower MZI output waveguides are distributed respectively to the 6 output channels in the two 3-channel AMMIs.

On the same SOI waveguide platform we have used previously $[\underline{3}-\underline{5}, \underline{8}, \underline{10}]$, the design parameters of the two AMMIs for the use of interleaved device were optimized for the mid-IR operation (Table 1). Compared to the work in [5], the channel spacing of a single 3-channel AMMI has been reduced from 30 to $26 \mathrm{~nm}$ so that the wavelength span of the 6-channel IAMMI having a channel spacing of $13 \mathrm{~nm}$ can fit in the effective wavelength range of the QCL-3.725-3.81 $\mu \mathrm{m}$.

The matching MZI for the interleaved device was designed using Eq. (5), which resulted in a single-mode MZI arm waveguide width of $1.2 \mu \mathrm{m}$ and an arm length difference of $\Delta L_{\mathrm{MZI}}=118.4 \mu \mathrm{m}$. For low insertion loss and wavelength independent operation, an optimized $1 \times 2 \mathrm{MMI}$ power splitter and a $2 \times 2 \mathrm{MMI}$ coupler with simulated insertion loss of about $0.1 \mathrm{~dB}$ for both were used in the MZI. To reduce the insertion loss of the MZI, we have used a sufficiently large bending radius $(R=50 \mu \mathrm{m})$ to have a negligible bending loss for the $400 \mathrm{~nm} / 180 \mathrm{~nm}$ rib waveguide in the mid-IR wavelength range. Also, the fewest joints between straight waveguides and bent waveguides were used in the arms so that the mode transition loss at the interface between the straight and bending waveguides were minimized. The two output branches from the MZI were connected to the inputs of AMMI1 and AMMI2 via adiabatic tapers so that the light from the MZI was maintained in the

Table 1. Design Parameters for the Two 3-Channel AMMIs

\begin{tabular}{lccc}
\hline$W_{\text {IO }}=1.2 \mu \mathrm{m}, W_{a}=28 \mu \mathrm{m}, W_{\text {AMMI }}=50 \mu \mathrm{m}, \theta=26.4^{\circ}$ \\
\hline AMMI1 & $L_{1}(\mu \mathrm{m})$ & $L_{3}(\mu \mathrm{m})$ & $L_{5}(\mu \mathrm{m})$ \\
& 5140 & 5082 & 5025 \\
AMMI2 & $L_{2}(\mu \mathrm{m})$ & $L_{4}(\mu \mathrm{m})$ & $L_{6}(\mu \mathrm{m})$ \\
& 5111 & 5053 & 4997 \\
\hline
\end{tabular}


waveguides' fundamental modes when propagating to the $28 \mu \mathrm{m}$ wide input waveguides of the AMMIs.

In consideration of effective coupling and normalization of light for insertion loss measurement, taper-grating units similar to those as used in $[8,10]$ were incorporated into the design. This unit is composed of a $1.2 \mu \mathrm{m}$-wide single-mode waveguide in connection with an adiabatic taper up to a $28 \mu \mathrm{m}$ wide waveguide, where surface gratings were fabricated for in/out-coupling of light via optical fibers. The input waveguide to the MZI is directly connected to one of the taper-grating units. The six $28 \mu \mathrm{m}$ wide output waveguides from the AMMIs were first connected to $1.2 \mu \mathrm{m}$ wide single-mode waveguides via adiabatic tapers that act as mode filters and then to six taper-grating units. A separate structure incorporating two of the taper-grating units connected back to back was used for normalization.

The designed interleaved AMMI devices were patterned on a high-resolution positive electron beam (e-beam) resist, ZEON ZEP520A, spun on a 6-inch SOI wafer with a silicon overlayer thickness of $400 \mathrm{~nm}$ and BOX thickness of $2 \mu \mathrm{m}$. The wafer was then written by an e-beam lithography system, JEOL JBX-9300FS. For this particular work, this system produced a stable e-beam spot size of $50 \mathrm{~nm}$ and was operated at an acceleration voltage of $100 \mathrm{kV}$. The wafer was patterned in stitching-error-free high-resolution mode within an area of $1 \mathrm{~mm} \times 1 \mathrm{~mm}$. When writing a large pattern, it achieved a stitching error of less than $20 \mathrm{~nm}$ and a positional accuracy of $1 \mathrm{~nm}$. After a single e-beam writing step and resist development, the pattern was transferred to the wafer using inductively coupled plasma (ICP) etching. The fabricated devices were air-cladded for testing. The microscopic images of the fabricated IAMMI are shown in Fig. 3. Stand-alone imbalanced MZIs were also fabricated to allow experimental analysis of their contribution to the device loss.

Figure 4 gives the measured spectral responses of the fabricated devices. The IAMMI device has an insertion
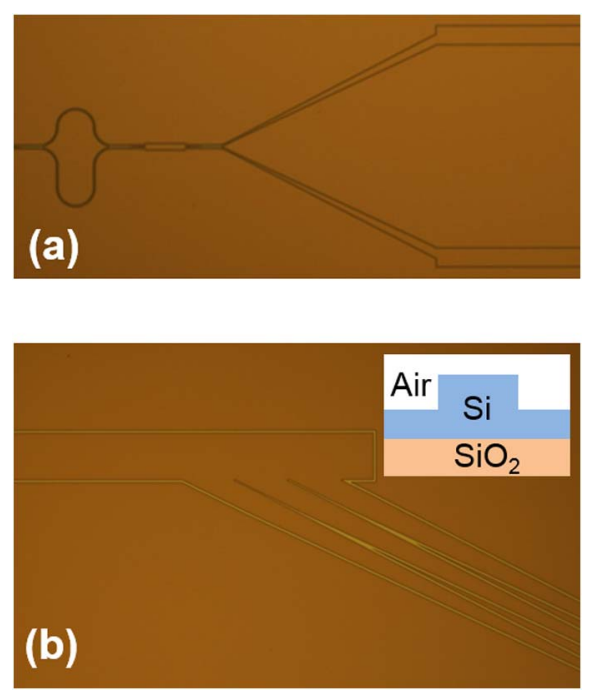

Fig. 3. Microscopic images of the fabricated IAMMI. (a) Section including the MZI and the AMMIs' inputs. (b) Output channels section with the inset showing the cross section of the waveguides.
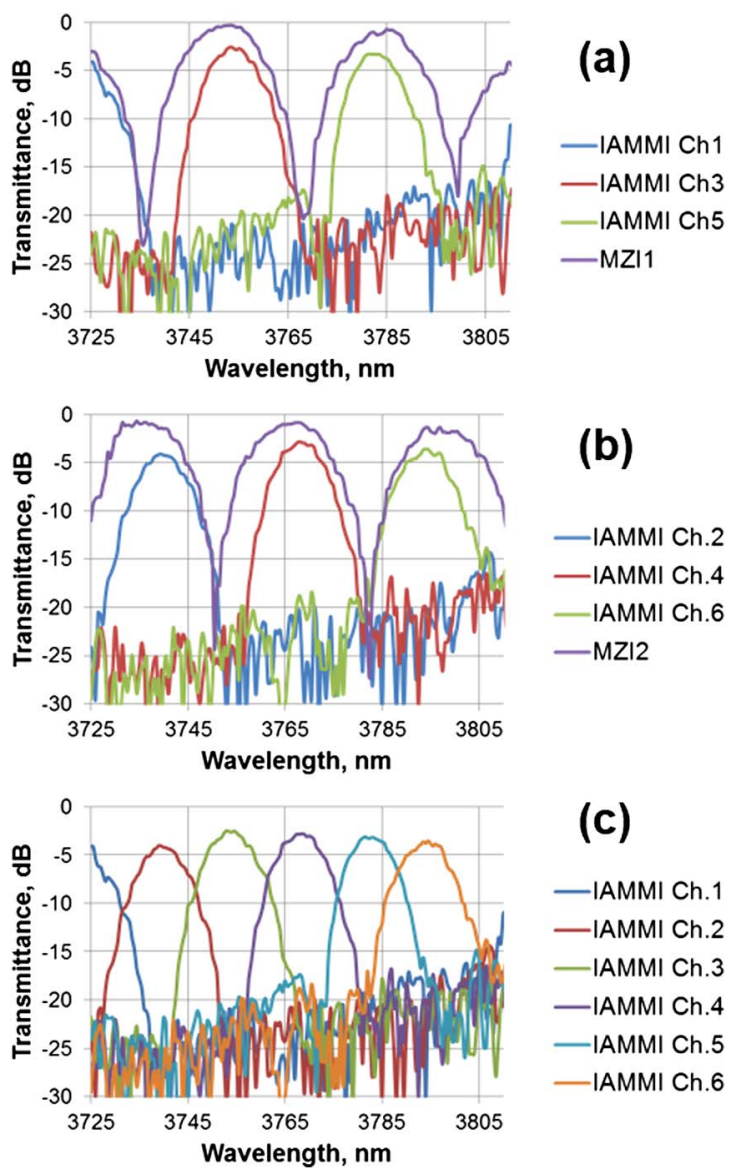

Fig. 4. Spectral responses of fabricated devices: (a) the 3-channels in AMMI1 of the IAMMI and the stand-alone MZI channel 1, (b) the 3-channels in AMMI2 of the IAMMI and the stand-alone MZI channel 2, and (c) the 6 channels of the IAMMI.

loss of 3-4 $\mathrm{dB}$ and a crosstalk of $-(15-18) \mathrm{dB}$ across the 6 channels. The stand-alone MZI's insertion loss was significantly reduced to be $<1 \mathrm{~dB}$ as compared to our previous experiment in the near-IR wavelength range [10]. It proves the effectiveness of the minimization of insertion loss in the design of the MZI mentioned above. The device's peak transmission power has a nonuniformity of about $1 \mathrm{~dB}$ across the 6 channels, with the maximum transmittance occurring in the center (channel 3 and 4) and the minimum at the edges (channel 1 and 6 ). This is mainly due to the increased mismatch of peak transmission wavelengths between the AMMI and the MZI from the center toward the edges of the transmission band. Indeed, the AMMI was designed to have equal wavelength spacing between the 6 channels. However, the imbalanced MZI's FSR is wavelength dependent, i.e., the spacings between the neighboring transmission peaks are not uniform. This wavelength dependency becomes more evident for wider wavelength bands. In our case, for the best matching of transmission peaks, the wavelength channel spacing of the AMMI was designed to match the average FSR of the MZI across the band. One potential improvement is to have each individual channel of the AMMIs' transmission peaks match the MZI's respectively, i.e., a restrict satisfaction of Eq. (5). Furthermore, Figs. $\underline{4(\mathrm{a})}$ and $\underline{4(\mathrm{~b})}$ show there 
is about $2 \mathrm{~dB}$ difference in insertion loss between the central channels of the IAMMI (channel 3 and 4) and the MZI at the peaks. This difference is smaller (by about $1 \mathrm{~dB}$ ) in the near-IR case [10]. As far as we are concerned, this is mainly due to the increased oxide loss for a 3.725$3.81 \mu \mathrm{m}$ wavelength range [3]. The crosstalk of the device deteriorates toward longer wavelengths. It is due to the increased noise floor level of the QCL laser rather than the nature of the device. The IAMMI reported here has the similar number of channels, insertion loss and crosstalk compared to previously reported AWGs and PCGs in the same wavelength range [3]. The IAMMI solution has a footprint advantage compared to the AWG and PCG solutions [3] and a fabrication tolerance advantage compared to compact resonator-based designs [10].

In conclusion, we have demonstrated a 6 -channel midIR IAMMI WDM structure on the SOI platform. The whole device requires only single-step lithography and etching for fabrication. An insertion loss of 3-4 dB and a crosstalk of -(15-18) dB was achieved. The WDM's channel spacing was halved and the channel count was doubled compared to the previously reported single mid-IR AMMI without significant increase of footprint. The performance can potentially be improved by using further optimized design.

This work has been supported by Goran Mashanovich's Royal Society Research Fellowship and UK Silicon Photonics Programme funded by EPSRC. The fabrication of the (de)multiplexers was carried out at the Southampton Nanofabrication Centre (SNC), University of Southampton, UK. Youfang Hu acknowledges funding from the State Key Laboratory of Advanced Optical Communication Systems Networks, China.

\section{References}

1. G. Z. Mashanovich, M. M. Milosevic, M. Nedeljkovic, N. Owens, B. Xiong, E.-J. Teo, and Y. Hu, Opt. Express 19, 7112 (2011).

2. M. M. Milosevic, M. Nedeljkovic, T.-B. Masaud, E. Jaberansary, H. M. H. Chong, N. G. Emerson, G. T. Reed, and G. Z. Mashanovich, Appl. Phys. Lett. 101, 121105 (2012).

3. M. Muneeb, X. Chen, P. Verheyen, S. Pathak, A. Malik, M. Nedeljkovic, J. Van Campenhout, G. Z. Mashanovich, and G. Roelkens, Opt. Express 21, 11659 (2013).

4. G. Roelkens, U. Dave, A. Gassenq, N. Hattasan, C. Hu, B. Kuyken, F. Leo, A. Malik, M. Muneeb, E. Ryckeboer, Z. Hens, R. Baets, Y. Shimura, F. Gencarelli, B. Vincent, R. Loo, J. Van Campenhout, L. Cerutti, J.-B. Rodriguez, E. Tournié, X. Chen, M. Nedeljkovic, G. Z. Mashanovich, S. Li, N. Healy, A. C. Peacock, X. Liu, R. Osgood, and W. J. Green, Opt. Mater. Express 3, 1523 (2013).

5. M. Nedeljkovic, A. Z. Kokhar, Y. Hu, X. Chen, J. S. Penades, S. Stankovic, H. M. H. Chong, D. J. Thomson, F. Y. Gardes, G. T. Reed, and G. Z. Mashanovich, Opt. Mater. Express 3, 1205 (2013).

6. E. M. P. Ryckeboer, A. Gassenq, M. Muneeb, N. Hattasan, S. Pathak, L. Cerutti, J.-B. Rodriguez, E. Tournie, W. Bogaerts, R. Baets, and G. Roelkens, Opt. Express 21, 6101 (2013).

7. M. N. Petrovich, N. V. Wheeler, N. K. Baddela, F. Poletti, E. Numkam Fokoua, J. R. Hayes, D. R. Gray, and D. J. Richardson, presented at the 14th International Conference on Transparent Optical Networks (ICTON), Coventry, July 2-5, 2012, pp. 1-4.

8. Y. Hu, R. M. Jenkins, F. Y. Gardes, E. D. Finlayson, G. Z. Mashanovich, and G. T. Reed, Opt. Lett. 36, 4488 (2011).

9. L. B. Soldano and E. C. M. Pennings, J. Lightwave Technol. 13, 615 (1995).

10. Y. Hu, F. Y. Gardes, D. J. Thomson, G. Z. Mashanovich, and G. T. Reed, Appl. Phys. Lett. 102, 251116 (2013). 